\title{
Subacromial-subdeltoid bursitis following COVID-19 vaccination: a case of shoulder injury related to vaccine administration (SIRVA)
}

\author{
Tatiane Cantarelli Rodrigues $^{1}$ (1) $\cdot$ Pedro Filgueiras Hidalgo ${ }^{2,3} \cdot$ Abdalla Youssef Skaf $^{1,4} \cdot$ Aline Serfaty $^{5,6}$
}

Received: 23 March 2021 / Revised: 25 April 2021 / Accepted: 26 April 2021 / Published online: 4 May 2021

(c) ISS 2021

\begin{abstract}
Vaccination injection site adverse reactions are usually mild and transient, and post-vaccination musculoskeletal symptoms, such as myalgia and arthralgia, are very common. Shoulder injury related to vaccine administration (SIRVA), defined as shoulder pain and limited range of motion occurring after the administration of a vaccine intended for intramuscular administration in the upper arm, is a well-established condition in the medical literature, yet underreported. In such cases, subacromial-subdeltoid bursitis may occur, leading to shoulder dysfunction and ongoing pain. Millions of doses of vaccines for the prevention of COVID-19 have been administered to adults worldwide during the pandemic. We report a case of subacromial-subdeltoid bursitis after COVID-19 vaccination, related to the unintentional injection of vaccine solution into the bursa resulting in a robust immune-mediated inflammatory reaction.
\end{abstract}

Keywords COVID-19 · SIRVA · Bursitis · Vaccine $\cdot$ Shoulder · MRI · Ultrasound

\section{Introduction}

Vaccination injection site adverse reactions are common, and symptoms are usually mild and transient [1-3], with pain, swelling, and redness at the site of injection being the

Tatiane Cantarelli Rodrigues

tcantarelli@gmail.com

Pedro Filgueiras Hidalgo

drpedrofilgueiras@gmail.com

Abdalla Youssef Skaf

abskaf@gmail.com

Aline Serfaty

alineserfaty@gmail.com

1 Department of Radiology, Hospital Do Coração (HCor) and Teleimagem, Rua Desembargador Eliseu Guilherme 53 7th floor, São Paulo, SP 04004-030, Brazil

2 Ortopedia São Marcos, Rua Raul Veiga, 35 Centro, Cabo Frio, RJ 28907-090, Brazil

3 Orthoclin, Rio de Janeiro, RJ, Brazil

4 ALTA Diagnostic Center (DASA Group), São Paulo, SP, Brazil

5 Medscanlagos Radiology, Rua Manoel Francisco Valentim, 57, Cabo Frio, RJ 28906220, Brazil

6 Universidade Federal Do Rio de Janeiro, Rio de Janeiro, RJ, Brazil most common clinical findings [3, 4]. Cases of shoulder injury related to vaccine administration (SIRVA), defined as shoulder pain and limited range of motion occurring after the administration of a vaccine intended for intramuscular administration in the upper arm, have been reported in the medical literature [2, 3, 5-8], and influenza is the most frequently vaccine reported $[1,3,9,10]$. Although subacromial-subdeltoid bursitis is a common health problem with a prevalence of approximately $1 \%$ in the general US population [11], its occurrence within the context of SIRVA is still underreported in the literature.

The relationship between vaccine injection into synovial tissue and inflammation of the structures underlying the deltoid muscle has been well established [3-9]. In this setting, bursitis, adhesive capsulitis, and glenohumeral synovitis may occur [3-9], with potential need for medical assistance due to severe prolonged pain and joint mobility restriction $[1,5]$. In the past 2 years, much has been studied about the multi-systemic involvement of COVID-19. However, potential adverse effects related to global COVID-19 vaccination still need to be further explored.

To our knowledge, this is the first case reported of postvaccination subacromial-subdeltoid bursitis after a COVID19 vaccination due to the unintentional injection of vaccine solution into the bursa, resulting into a robust immunemediated inflammatory reaction. Raising awareness of this 
underreported potential complication is crucial, as millions of people receive the injection with the ongoing roll-out of COVID-19 vaccination programs. The large number of vaccine doses administered daily [12] can lead to a significant increase in workload and rushed procedures, and as a consequence, the risk of inappropriately injected vaccines can also rise.

\section{Case report}

A 61-year-old woman presenting with excruciating pain and tenderness at the injection site in her right shoulder within 30 min after receiving her first shot of COVID-19 vaccine (Oxford-AstraZeneca, Serum Institute of India). She voiced concern about vaccine administration, specifically that the injection had been administered "too high" in the arm. Informed consent was obtained from the subject described in this report.

Photographs of the patient while receiving the vaccine were taken and confirmation of the incorrect technique used was noticed. The injection was administered at the level of two fingerbreadths of the lateral border of the acromion, which is considered higher than the recommendation (Fig. 1).

Initial treatment included ice pack, topical diclofenac cream, and a combination of caffeine, carisoprodol, sodium diclofenac, and paracetamol for 5 days from the vaccine injection.

Eight weeks after the vaccination, the patient was still experiencing persistent pain in the right shoulder and reduced range of motion with inability to perform daily activities. Upon physical examination, there was mild swelling of the upper arm and localized shoulder tenderness around the top of the humeral head and deltoid. Active and passive ranges of motion were evaluated through orthopedic maneuvers. The strength of the rotator cuff muscles and the normal passive range of motion were preserved. The active range of motion in the shoulder was limited with active painful $\operatorname{arc}\left(45^{\circ}\right.$ of abduction, $20^{\circ}$ of extension, $45^{\circ}$ of flexion). Neer's, Hawkins', Yocum's, and O'Brien's tests were positive, while Jobe's and Patte's tests were negative. Gerber's test was not performed due to pain complaints. Physical neurovascular exam was normal.

Routine X-rays of the shoulder were performed and did not provide helpful diagnostic information (Fig. 2a). Shoulder MRI and ultrasound 8 weeks after vaccination supported a diagnosis of subacromial-subdeltoid bursitis and rotator cuff tendinopathy (Fig. 2b-f). The fluid-filled subacromialsubdeltoid bursa was greater than is commonly seen for overuse bursitis caused by mechanical stress. The correlation with hypertrophic synovial tissue raised the hypothesis of an underlying inflammatory cause.

The patient started prednisone oral treatment, vitamin D supplementation, and a physical therapy regimen to
Fig. 1 Photograph of the patient receiving her first dose of the COVID-19 vaccine (OxfordAstraZeneca, Serum Institute of India) in Brazil's vaccination program into the upper third of the right deltoid muscle, which is considered higher than the recommended area (red circle)
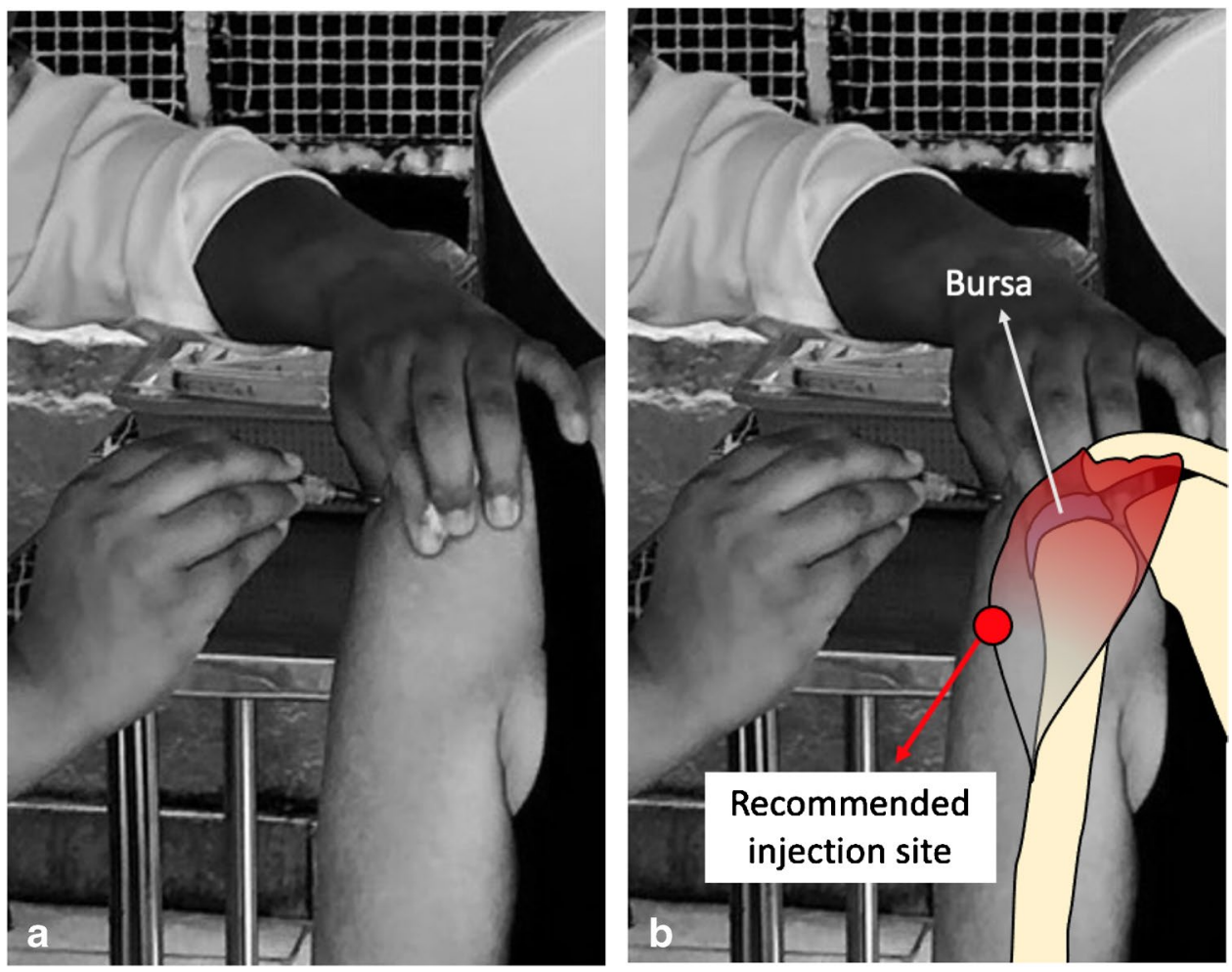

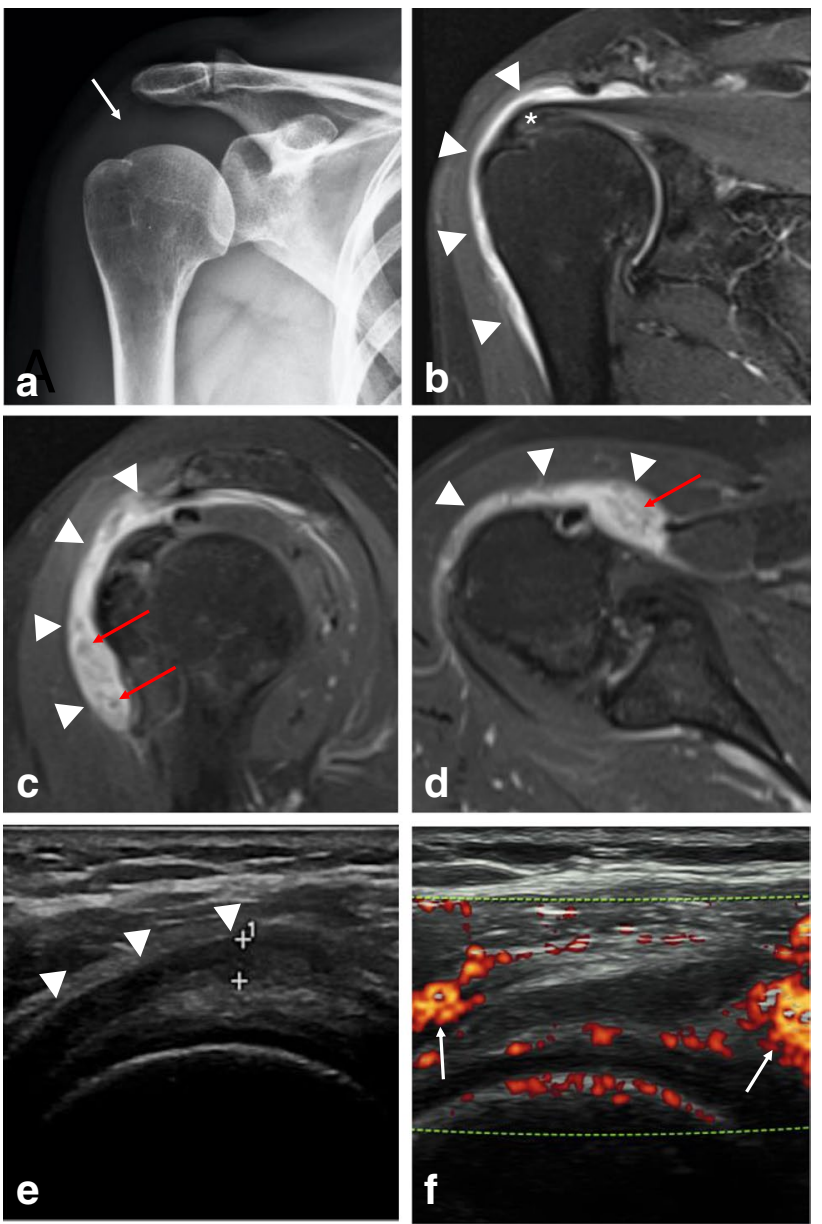

Fig. 2 Subacromial-subdeltoid bursitis on the right shoulder 8 weeks after the COVID-19 vaccination. (a) Radiograph of the right shoulder in AP view shows soft tissue density of fat pad adjacent to the subacromial-subdeltoid bursa (arrow). The (b) coronal and (c) sagittal T2-weighted fat-suppressed MR images, and the (d) axial proton density-weighted axial image demonstrating the lateral to medial extension of fluid in the subacromial-subdeltoid bursa (arrowheads) with synovial hypertrophy (red arrows). Supraspinatus tendinosis is seen with the signal-alteration (asterisk). Ultrasound of the right shoulder (e) demonstrates hypoechoic fluid accumulation within the subacromial-subdeltoid bursa (arrowheads) more than $3.0 \mathrm{~mm}$. Thickening of the bursal walls is also noted (arrow). (f) Longitudinal power Doppler sonogram shows moderate increase of surrounding perfusion in adjacent soft tissue (arrows)

preserve the range of motion, in an attempt at minimizing potential complications, such as adhesive capsulitis.

As past medical history, the patient had diagnosed hypothyroidism due to Hashimoto's thyroiditis, also known as chronic lymphocytic thyroiditis, treated with sodium levothyroxine for 21 years. No medical history of inflammatory arthropathy or previous COVID-19 infection was reported.

\section{Discussion}

A diagnosis of shoulder injury related to vaccine administration (SIRVA) was made due to the rapid onset of pain after intramuscular vaccine administration in a patient with no prior history of chronic pain or inflammatory diseases in the affected shoulder combined with findings consistent with a local immune-mediated inflammatory reaction. The key to recognizing SIRVA is that the pain usually begins within $48 \mathrm{~h}$ of vaccine administration, which may persist for months. Symptoms include associated weakness and impaired mobility, without improvement with over-thecounter analgesics [5].

Millions of doses of vaccines for the prevention of coronavirus disease 2019 have been administered to adults around the world during the COVID-19 pandemic [12]. Immunization using the COVID-19 vaccine AstraZeneca of individuals 18 years of age and older has been done since emergency use authorization for the shot on December 30, 2020, in the UK by the UK Medicines and Healthcare products Regulatory Agency (MHRA) [13]. AstraZeneca's COVID-19 vaccine has been approved for emergency supply in Brazil on January 17, 2021, by the Brazilian Health Regulatory Agency (ANVISA) [14]. This vaccine should be given in two separate doses by an authorized vaccinator as an intramuscular injection only, preferably into the deltoid muscle[15].

One dose of COVID-19 vaccine contains $5 \times 10$ [10] viral particles in $0.5 \mathrm{ml}$ of solution. The active substance of COVID-19 vaccine AstraZeneca is a monovalent vaccine composed of a single recombinant, replicationdeficient chimpanzee adenovirus (ChAdOx1) vector, that codes for the S glycoprotein of SARS-CoV-2 (ChAdOx1-S [recombinant]) [15].

The most common side effects reported with COVID19 vaccine AstraZeneca, which may affect more than 1 in 10 people, were tenderness $(63.7 \%)$ and pain $(54.2 \%)$ at the injection site. Myalgia (44.0\%) and arthralgia (26.4\%) were common musculoskeletal side effects reported. During the clinical trials, a majority of adverse reactions reported were mild to moderate in nature and resolved within a few days, with some lasting for a week after vaccination. When compared to the first dose, adverse reactions reported after the second dose were milder and less frequent [15].

Our patient developed shoulder pain shortly after vaccination, possibly due to her vaccine being administered inadvertently higher than recommended (Fig. 3) [4, 7]. Atanasoff and colleagues, in a retrospective study about SIRVA, reported that in $93 \%$ of the cases, shoulder pain occurred less than $24 \mathrm{~h}$ after vaccination and immediately following injection in 54\% [2]. 


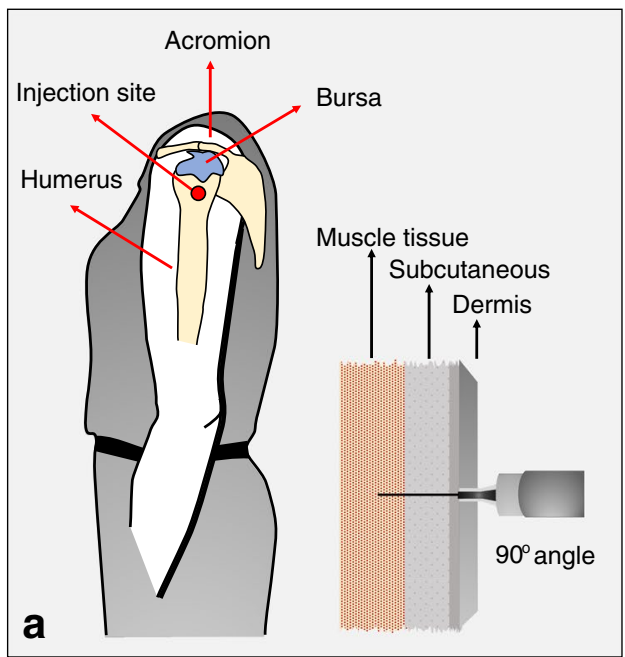

Fig. 3 a Deltoid muscle is recommended for routine intramuscular vaccination for adults. The safest anatomical site in adults of both sexes would be approximately $7-13 \mathrm{~cm}$ (three fingerbreadths) below the mid-acromion, anatomically midway between the acromion and the deltoid tuberosity (red circle). Intramuscular injections are administered at an angle between 72 to $90^{\circ}$ to the skin. A $2.5-\mathrm{cm}$ (1-in.)

This adverse reaction can also be caused when the injection is too deep, going through the muscle and into the bursa $[4,7,8,10]$. Bodor and Montalvo found that the subdeltoid bursa extended from 3.0 to $6.0 \mathrm{~cm}$ beyond the lateral border of the acromion at a depth from 0.8 to 1.6 $\mathrm{cm}$ [7] which could be easily reached by the $2.5-\mathrm{cm}$ (1-in.) needle used for the injection in our patient. Guidelines for proper administration, including explicit instruction to avoid the upper third of the deltoid, would help to reduce the risk of penetrating the bursa during vaccine injections [2]. Inappropriate technique could potentially reduce vaccine efficacy or increase the risk for local adverse reactions $[4,10]$.

The patient demonstrated reduced active range of motion and ongoing shoulder pain that lasted for at least 8 weeks after receiving her first shot of the COVID-19 vaccine. The most common findings upon examination of patients with SIRVA are an active painful arc, with the severity and duration of shoulder dysfunction varying among patients, which may ultimately last for years [2].

Imaging studies from our patient supported a diagnosis of subacromial subdeltoid bursitis and rotator cuff tendinopathy. Subacromial subdeltoid bursitis is considered a known but rare cause of vaccine-related morbidity [7]. In a study of influenza vaccination, the analysis found an increased risk of 7.8 for subdeltoid bursitis with the onset of symptoms within 3 days after vaccination [3]. MRI findings usually include fluid collections in the deep deltoid or overlying the rotator cuff tendons, bursitis, fluid "greater than typically seen" within the bursa, tendonitis, and rotator cuff tears [2].

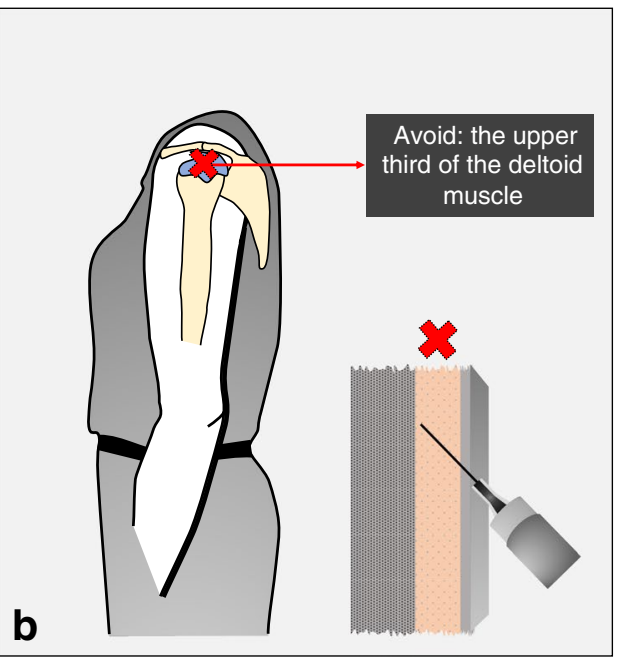

needle depth is appropriate for most patients; it is long enough to reach the muscle mass and prevent overpenetration. b The upper third of the deltoid muscle should not be used for vaccine injections. An angle close to $45^{\circ}$ is not recommended for intramuscular vaccinations, as it can lead to the application of the vaccine in the dermis or subcutaneous

Shoulder injury originating from vaccination is usually greater than would be expected from a simple needle trauma. A robust synovial inflammation may be seen and septic arthritis is a differential diagnosis and should be excluded [8]. Non-invasive imaging studies, such as MRI or ultrasonography, can be helpful in differentiating this condition and other more common pathologies (e.g., arthritis, rotator cuff tears, calcific tendonitis).

In cases in which severe local or systemic signs or symptoms are present and SIRVA is suspected, non-invasive imaging evaluation may be required immediately to rule out inflammatory conditions or infection and to determine the level and type of damage, especially when the symptoms persist and additional invasive procedures, such as steroid injections, might be considered [2].

When an individual is vaccinated, vaccine antigen-antibody interaction reaction caused by vaccination at the local site is expected to be transient and resolve as the antigen is cleared from the soft tissues over a period of several days [2]. However, in cases in which vaccine solution is inadvertently injected into the synovial space of the shoulder, pre-existing antibodies in the synovial tissues from previous infection or past-vaccination, may lead to a more prolonged inflammatory response [2].

Some authors [2] believe that this type of phenomenon is a result from injection of a vaccine antigen from which a person has previously been sensitized, rather than due to a specific vaccine. Although COVID-19 is a new infectious disease and the population is receiving COVID-19 vaccines for the first time, and although the patient had not 
been previously directly exposed to the coronavirus, it is unlikely that an adult would not have had previous exposure to an adenovirus in the past, which is in fact a component of COVID-19 vaccine AstraZeneca.

The majority of patients may have persistent symptoms including pain and a limited range of motion [2, 5]. In many cases, resting and anti-inflammatory medications can lead to symptom resolution within a few weeks [3]; however, in some cases, an injection of a corticosteroid over time may be required[2, 5]. Surgical intervention can be performed in refractory cases $[2,10,16]$.

The diagnosis of SIRVA should be considered in patients with established symptoms of shoulder pain following a vaccination. Vaccine administration in the upper third of the deltoid area can have long-lasting consequences unrelated to the specific vaccine administered. Awareness of the potential for inducing a prolonged immune-mediated inflammatory reaction if a vaccine antigen is injected into synovial tissue structures underlying the deltoid muscle should be emphasized, as well as careful consideration given of proper injection technique when administering shoulder intramuscular vaccinations.

SIRVA is well established in the medical literature [5, 17]; however, it is still an underreported condition. To our knowledge, this is the first case report with a causal relationship following a COVID19 vaccination. SIRVA is a preventable condition if the vaccine is administered properly. Millions of vaccines for COVID-19 are being administered daily to adults worldwide due to the pandemic; thus, the number of vaccines injected inappropriately can likewise increase. Shoulder injuries should be considered one of the potential adverse effects of the vaccination.

\section{Declarations}

Conflict of Interest The authors declare no competing interests.

\section{References}

1. Martín Arias LH, Sanz Fadrique R, Sáinz Gil M, SalgueiroVazquez ME. Risk of bursitis and other injuries and dysfunctions of the shoulder following vaccinations. Vaccine. 2017;35(37):4870-6. https://doi.org/10.1016/j.vaccine.2017.07.055.

2. Atanasoff S, Ryan T, Lightfoot R, Johann-Liang R. Shoulder injury related to vaccine administration (SIRVA). Vaccine. 2010;28(51):8049-52. https://doi.org/10.1016/j.vaccine.2010.10.005.
3. Batra S, Page B. Shoulder injury related to vaccine administration: case series of an emerging occupational health concern. Workplace Health Saf. 2021;69(2):68-72. https://doi.org/10.1177/ 2165079920952765.

4. Hexter AT, Gee E, Sandher D. Management of glenohumeral synovitis secondary to influenza vaccination. Shoulder Elbow. 2015;7(2):100-3. https://doi.org/10.1177/1758573214560258.

5. Bancsi A, Houle SKD, Grindrod KA. Getting it in the right spot: shoulder injury related to vaccine administration (SIRVA) and other injection site events. Can Pharm J (Ott). 2018;151(5):295-9. https://doi.org/10.1177/1715163518790771.

6. Barnes MG, Ledford C, Hogan KA. "needling" problem: shoulder injury related to vaccine administration. J Am Board Fam Med. 2012;25(6):919-22. https://doi.org/10.3122/jabfm.2012.06. 110334.

7. Bodor M, Montalvo E. Vaccination-related shoulder dysfunction. Vaccine. 2007;25(4):585-7. https://doi.org/10.1016/j.vacci ne.2006.08.034.

8. Cross GB, Moghaddas J, Buttery J, Ayoub S, Korman TM. Don’t aim too high: avoiding shoulder injury related to vaccine administration. Aust Fam Physician. 2016;45(5):303-6.

9. Behrens RH, Patel V. Avoiding shoulder injury from intramuscular vaccines. Lancet. 2021;397(10273):471. https://doi.org/10. 1016/S0140-6736(21)00192-6.

10. Hesse EM, Navarro RA, Daley MF, et al. Risk for subdeltoid bursitis after influenza vaccination: a population-based cohort study. Ann Intern Med. 2020;173(4):253-61. https://doi.org/10.7326/ M19-3176.

11. Walker-Bone K, Palmer KT, Reading I, Coggon D, Cooper C. Prevalence and impact of musculoskeletal disorders of the upper limb in the general population. Arthritis Rheum. 2004;51(4):64251. https://doi.org/10.1002/art.20535.

12. Ritchie H, Ortiz-Ospina E, Beltekian D, Mathieu E, al e. Our world in data: coronavirus (COVID-19) vaccinations . Accessed March 16th, 2021, 2021. https://ourworldindata.org/covid-vacci nations

13. 2021 Regulatory approval of COVID-19 Vaccine AstraZeneca (2020).

14. Relatorio: bases técnicas para decisão do uso emergencial, em caráter experimentar de vacinas contra a COVID 19 (2021).

15. Agency EM. COVID-19 vaccine AstraZeneca - product information as approved by the CHMP on 29 January 2021, pending endorsement by the European Commission. Accessed March 16th, 2021. https://www.ema.europa.eu/en/documents/product-infor mation/covid-19-vaccine-astrazeneca-product-information-appro ved-chmp-29-january-2021-pending-endorsement_en.pdf

16. Bliddal H, Torp-Pedersen S, Falk-Riecke B, Bartels E. [Bursitis after vaccination in the shoulder region]. Ugeskr Laeger. Oct 2017;179(43)

17. Bancsi A, Houle SKD, Grindrod KA. Shoulder injury related to vaccine administration and other injection site events. Can Fam Physician. 01 2019;65(1):40-42.

Publisher's note Springer Nature remains neutral with regard to jurisdictional claims in published maps and institutional affiliations. 